\title{
Channel Modeling for Overhead Line Equipment for Train Communication
}

\author{
Waled Gheth ${ }^{1}$, Khaled M. Rabie ${ }^{1}$, Bamidele Adebisi ${ }^{1}$, Muhammad Ijaz ${ }^{1}$ and Georgina D. Harris ${ }^{2}$ \\ ${ }^{1}$ Department of Engineering, Manchester Metropolitan University, UK \\ ${ }^{2}$ School of Science, Engineering and Environment, University of Salford, UK \\ Emails:\{w.gheth, k.rabie, b.adebisi,m.ijaz, g.harris\}@mmu.ac.uk; g.d.harris14@salford.ac.uk
}

\begin{abstract}
The demand for high-speed data access to railway infrastructure and internet broadband data in railway is increasing due to the high density of the trains and passengers. Currently, communication access in trains is based on radio frequency (RF) wireless access networks that are slow and insufficient for the demands of the high-speed railway (HSR) and its customers. However, performance, service attributes, frequency band, and industrial support should be considered for the selection of a suitable communication system that can fulfill the requirements of HSR operation. This paper investigates overhead line equipment (OLE) as access network connecting trains to the backbone communication networks. The ABCD transmission model is used to represent the transfer function of the OLE channel. It was shown that transmission over OLE is affected by the frequency and link distance. The simulation results also show that the channel gain of the OLE channel attenuated faster at higher speeds compared to train movement at lower speeds.
\end{abstract}

Index Terms-Channel modeling, electrification system, high speed railway, overhead line equipment, reliable communication system.

\section{INTRODUCTION}

$\mathbf{T}$ HE overhead line equipment (OLE) refers to the wires found along electrified railways which are used to transmit electricity to the trains so they could move. The supply of electrical power to the trains is divided into four main steps. First, power generation where the electricity is generated at the power stations; then at the transmission stage the power is transmitted to the overhead transmission lines of the national grid at very high voltage $(400 \mathrm{kV})$. After that, the feeder substations take the power from the national grid and convert it to $25 \mathrm{kV}$ to be transmitted to the catenary system [1]. Electricity is then collected from a contact wire by a panto-graph or a current collector which is attached to the roof of the running train. As the electrical power is something that trains must have access at all times, the contact wire overlaps with the next wire so that the panto-graph slides smoothly from one to the other [2].

OLE falls under the medium-voltage transmission lines as it carries power electricity at $25 \mathrm{kV}$. Several studies have discussed the medium-voltage power lines as a means of communication. The studies showed that the mediumvoltage power line communication (MV-PLC) can provide reliable transmission services and is regarded as the key technology for the last-mile communication [3], [4]. The use of MV-PLC has gained plenty of research interests since it represents a cost-effective solution for communication [5]. Monitoring systems, network management optimization and operational services are the common applications of the MVPLC technology [6]. The main advantage of this communication technique is that it uses the pre-installed medium-voltage power lines which means that the communication system is readily available.

As a result of the developments of this technology and for performance evaluation purposes, channel models that can represent the transfer function of the PLC channel are required. However, different channel models are represented in the literature. The multi-path and two-port network are the most relevant models to the PLC channel and they can accurately represent its transfer function for performance analysis [7]-[9]. In the former, the transfer function of the PLC channel is represented by three factors: attenuation, delay, and weighting. This model can offer the possibility to investigate several network topologies and examine their effect on the performance of the PLC system by means of simulations [7]. On the other hand, the two-port network model uses the transmission lines theory (TLT) for a two-port network to calculate the channel transfer function (CTF) of the PLC system. This can be easily achieved by simplifying and deriving the equivalent circuit of the PLC network and finding the relationship between the output and input values [8]. Different circuit parameters are considered in this model such as the number of branches in the circuit, branch length, loads impedance, etc.

OLE has always been considered as a means of railway electrification and, to the best of the authors' knowledge, no study in the literature has considered this medium for communication. Therefore, the main contributions of this work are as follows.

- Developing the two-port network model to represent the CTF of the catenary system of the railway electrification by using the TLT.

- In order to implement this model, we simplified the train electrification system and derived an equivalent circuit for the whole system. Different movement equations that consider the acceleration and deceleration of the train are used to update the train position which represents the length of the transmission line.

The rest of the paper is organized as follows. The general 


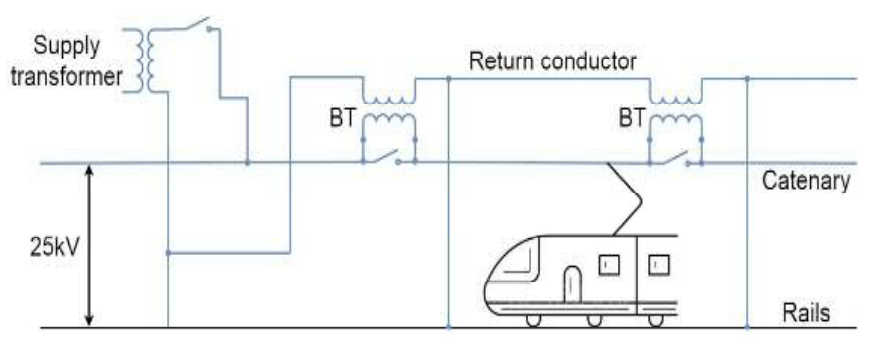

Fig. 1: Classical single-phase $1 \times 25 \mathrm{kV}$ feeding configuration with BT.

structure of the electrification system is introduced in Section II. The equivalent circuit of the OLE is also derived in this section. In Section III, the TLT is implemented in order to calculate the OLE transfer function based on actual parameters of the electrification system. Discussions of the simulation results are presented in Section IV. Finally, Section V draws the main conclusions of this paper.

\section{Overhead Line Equipment System}

In this section, the train electrification system is described and the equivalent circuit of the catenary system is derived.

Fig. 1 shows the classical single-phase $1 \times 25 \mathrm{kV}$ feeding configuration with the booster transformer (BT) feeding which is considered in this paper. The single-phase $1 \times 25 \mathrm{kV}$ is the standard railway electrification system used in the UK and many other countries for the OLE [10]. Each rail substation is fed by the utility grid through a power transformer where the primary of the transformer is connected to the utility grid and the secondary side is connected to the feeder station [11]. Direct, auto-transformer (AT), and BT feedings are the three basic feeding configurations utilized for providing electricity to trains in the railway sections [12], [13]. In this study, we only consider the BT feeding configuration as it is the feeding method used in the UK electrification system, as demonstrated in Fig. 1. This feeding configuration is used to tackle rail-to-earth leakage which is the main drawback of the direct feeding configuration [14].

\section{A. General Structure of the BT Feeding}

BTs are introduced to eliminate the interference with the communication lines that are used close to the track which is caused by the current leakage through the ground [15]. The connection of the BTs is achieved by two different approaches, either by connecting the primary to the contact wire of the catenary system and the secondary is connected directly to the rails or by connecting the secondary to a return conductor [16]. In the British railway electrification system, the leakage of the current is reduced by connecting the BTs between the contact wire and the return conductor. These BTs are normally located along the contact wire at almost every $3 \mathrm{~km}$ and they consist of the primary and secondary windings of unity turn-ratio. The current flows through the primary side of the BT that is connected in series with the contact wire of the catenary system. The flow of current in the primary winding induces a voltage in the winding of the

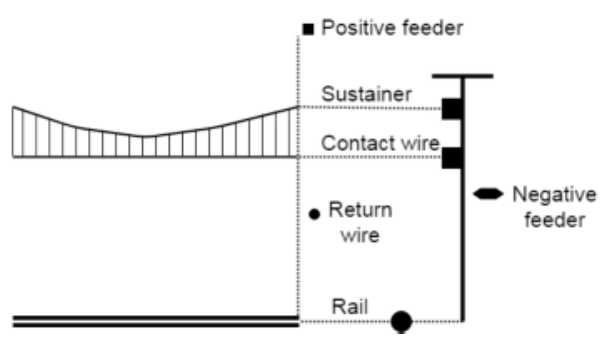

Fig. 2: Typical conductor distribution.

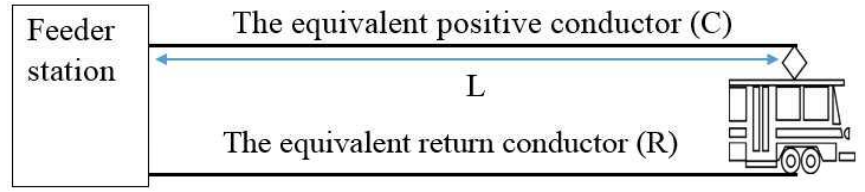

Fig. 3: A simplified conductors distribution.

secondary side of the BT. In order to have the same amount of primary current flowing in the secondary side, this induced voltage forces the return current to flow through the return feeder connected to the secondary winding of the BT, thus no current flows through the rails [16].

\section{B. BT Feeding Equivalent Circuit}

The catenary system is either equipped with one or two active conductors for $1 \times 25 \mathrm{kV}$ and $2 \times 25 \mathrm{kV}$, respectively. The catenary systems usually contain multiple physical conductors. These conductors can be divided into three different groups, namely positive, negative and ground wires [17]. Fig. 2 shows the typical configuration of the mono-voltage catenary of an AC high-speed railway line. While there is only one negative wire that represents the negative feeder, the positive group is represented by three wires, namely, the contact wire, the sustainer wire, and the positive feeder. The ground group also contains three conductors: the rail, the collector wire, and the return conductor [17]. The representation of the conductors of the same group can be reduced to one conductor by assuming that each conductor group has the same voltage. Furthermore, the current of the equivalent conductor of each group is equal to the sum of the currents of the physical conductors [16]. Fig. 3 presents a simplified conductors distribution. In order to implement the TLT and to simplify the feeding system, the BTs connected between the equivalent positive and return conductors have to be considered. As mentioned above, the primary side of the BT is connected to the positive conductor and the secondary side is connected to the return conductor. The BT has a leakage flux at both of its windings that can give leakage reactance $X_{1}$ and $X_{2}$. Furthermore, both windings possess resistances $R_{1}$ and $R_{2}$, as shown in Fig. 4 .

The impedance at the primary and secondary windings can be represented respectively by

$$
\begin{aligned}
& Z_{1}=R_{1}+X_{1} \\
& Z_{2}=R_{2}+X_{2} .
\end{aligned}
$$




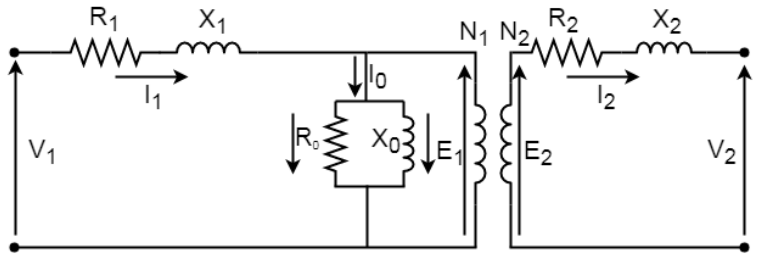

Fig. 4: Equivalent circuit of the BT.

Now, to simplify the equivalent circuit of the BT, the secondary side parameters can be referred to the primary side using

$$
\grave{Z}_{2}=Z_{2} \times n^{2}
$$

where $n$ is the ratio between the winding turns number of the primary and secondary sides, which is equal to one, as the $\mathrm{BT}$ is a $1: 1$ ratio transformer. The exciting current, which is drawn by $X_{0}$ and $R_{0}$, is very small and thus the excitation of this parallel $X_{0}$ and $R_{0}$ can be neglected. This means that the impedance of the primary side can be defined as

$$
Z_{e q}=Z_{1}+\grave{Z}_{2}
$$

Therefore, the equivalent circuit of the BT becomes as it appears below

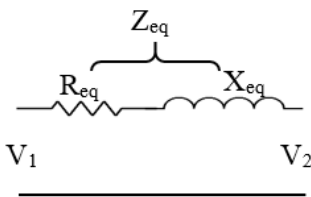

The $Z_{e q}$ of the BT is in series connection with the contact wire so it can be added to the contact wire impedance. Taking in consideration that trains can be represented by impedance model [18] and assuming that only one train is fed by a substation, the equivalent circuit of this feeding system can be simplified as

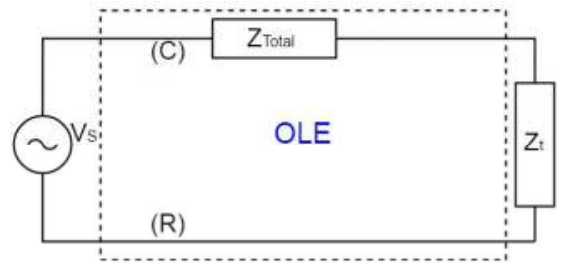

Now, the TLT can be implemented for the OLE. The OLE can be represented by a distributed network, which is a per-length-unit network, using its four parameters: the resistance $(\mathrm{R})$, the inductance $(\mathrm{L})$, the capacitance $(\mathrm{C})$ and the conductance $(\mathrm{G})$. This distributed network can be represented by a two-port network which is fully explained in the following section.

\section{OLE TRANSFER FUNCTION}

In this section, we study the transfer function of the OLE channel by implementing the ABCD matrix based on theoretical considerations.

The ABCD matrix model is widely used to represent the CTF of the PLC [8]. The main idea of this model is to use TLT for the two-port networks to define the relationship between the output and input values of a network as shown in Fig. 5.

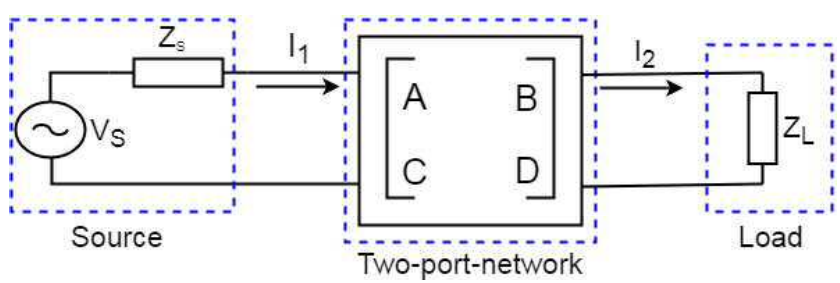

Fig. 5: A two-port network connected to a voltage source and a load.

The lumped network where the whole values of the primary parameters R, L, C and G of the OLE are lumped in one section and it can be represented by the following figure

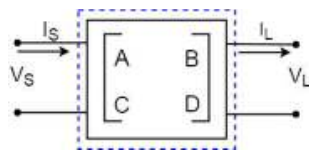

The relationship between the output and input values for this network is straightforwardly expressed as

$$
\begin{aligned}
& V_{S}=A \times V_{L}+B \times I_{L}, \\
& I_{S}=C \times V_{L}+D \times I_{L},
\end{aligned}
$$

where $V_{S}$ and $I_{S}$ are the sending voltage and current, respectively and $V_{L}$ and $I_{L}$ are the receiving voltage and current. A, B, C and D are properly selected constants known as the transmission parameters. These constants are utilized for the electrical networks analysis and studding the performance of the input and output of the transmission networks. However, for easy calculations, equations (5) and (6) can be given in a form of a matrix as

$$
\left[\begin{array}{l}
V_{S} \\
I_{S}
\end{array}\right]=\left[\begin{array}{ll}
A & B \\
C & D
\end{array}\right]\left[\begin{array}{l}
V_{L} \\
I_{L}
\end{array}\right]
$$

It is easy to derive the transfer function in the circuit of Fig. 5 using the ABCD matrix as

$$
H=\frac{V_{L}}{V_{S}}=\frac{Z_{L}}{A Z_{L}+B+C Z_{L} Z_{S}+D Z_{S}} .
$$

Also the input impedance can be easily calculated as

$$
Z_{S}=\frac{V_{S}}{I_{S}}=\frac{A Z_{L}+B}{C Z_{L}+D}
$$

The ABCD matrix is based on the characteristics of the OLE that are characterized by their propagation factor $(\gamma)$ 
and characteristic impedance $\left(Z_{c}\right)$ [8]. However, per-unitlength $\mathrm{R}, \mathrm{L}, \mathrm{C}$ and $\mathrm{G}$ calculations/measurements of the OLE are needed in order to calculate these two factors. Hence, the two secondary constants $\gamma$ and $Z_{c}$ of the OLE can be expressed as functions in the primary constants R, L, C and $\mathrm{G}$ of the OLE as

$$
\begin{gathered}
Z_{C}=\sqrt{\frac{R+j \omega L}{G+j \omega C}}, \\
\gamma=\sqrt{(R+j \omega L)(G+j \omega C)} .
\end{gathered}
$$

The effective per-unit-length $\mathrm{AC}$ resistance $(\Omega / m)$ of the OLE with length $l$ and cross-section area $A$ is equal to the direct-current resistance $\left(R_{d c}\right)$, which can be calculated as $R=\rho l / A$, where $\rho$ is the resistivity of the conductor material.

The per-unit-length of the OLE inductance $(H / m)$ is a combination of internal inductance $\left(L_{i n t}\right)$ and external inductance $\left(L_{e x t}\right)$. The internal inductance is due to internal flux linkages and the external inductance is caused by the external ones. However, the per-unit-length inductance is given by

$$
L=L_{i n t}+L_{\text {ext }},
$$

where $L_{i n t}=\frac{\mu}{8 \pi}$ and $L_{e x t}=\frac{\mu}{2 \pi} \times \ln \left(\frac{D}{r}\right)$, by substituting $L_{\text {int }}$ and $L_{\text {ext }}$ values into (12), we get the total inductance as

$$
L=\frac{\mu}{2 \pi} \times\left(\frac{1}{4}+\ln \left(\frac{D}{r}\right)\right),
$$

where $\mu$ is the permittivity of the conductor, $D$ is the distance between the conductors and $r$ is the radius of the conductor.

The per-unit-length capacitance $(F / m)$ can be calculated

$$
C=\frac{2 \pi \epsilon_{0} \epsilon_{r}}{\ln \left(\frac{D}{r}\right)}
$$

where $\epsilon_{0}$ represents the free space permittivity and $\epsilon_{r}$ depicts the relative permittivity of the medium.

As the parallel leakage of current is too small, the per unit length conductance $(S / m)$ can be neglected. However, the geometric mean distance (GMD) and geometric mean radius (GMR) of an irregular conductor are important for the primary parameters R, L, C and G calculation [19].

Hence, the ABCD matrix for a known transmission line length $l$, characteristic impedance $Z_{C}$ and propagation constant $\gamma$ is given by

$$
\left[\begin{array}{cc}
A & B \\
C & D
\end{array}\right]=\left[\begin{array}{cc}
\cosh (\gamma l) & Z_{c} \cdot \sinh (\gamma l) \\
\frac{1}{Z_{c}} \cdot \sinh (\gamma l) & \cosh (\gamma l)
\end{array}\right]
$$

The CTF of the OLE can be easily calculated by substituting the $A, B, C$ and $D$ values from (15) into (8).

Furthermore, the length of the transmission line changes according to the train movement. It increases if the train moves away from the substation and decreases when the

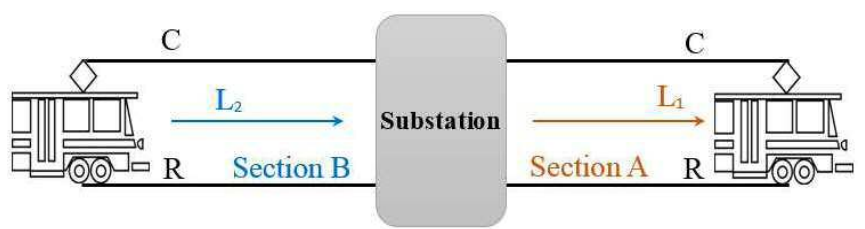

Fig. 6: Sections A and B of the railway under considerations.

train moves towards the substation. Therefore, the movement equations that consider the acceleration and deceleration of the train are crucial to update the length of the transmission line by updating the position of the train in the proposed model. The following equations are used in this model to update the train speed and position as

$$
\begin{gathered}
V=\frac{d}{t}, \\
V_{f}=V_{i}+a \times d t, \\
a=\frac{V_{f}-V_{i}}{t}, \\
l_{f}=l_{i}+V_{i}+\left(0.5 \times a \times d t^{2}\right),
\end{gathered}
$$

where $V_{i}$ and $V_{f}$ are the initial and final speeds of the train during the acceleration, $a$ is the acceleration factor, $\mathrm{d} t$ represents the step of the time, $t$ denotes the acceleration time, $l_{i}$ and $l_{f}$ are the length of the transmission line before and after the update.

\section{AnAlytical Results}

This section presents some simulation results of the CTF of the proposed OLE.

Different channel scenarios are involved to study the channel of the OLE and to explore the effect of different channel parameters (e.g., the channel length and the transmission frequency) on the performance of the transmission. It is assumed that a railway substation feeds two railway sections $\mathrm{A}$ and $\mathrm{B}$. The transmitter device, which is installed at the substation, sends data to both sections with one train moves in each section as shown in Fig. 6. The length of the OLE channel is defined as the distance between the transmitter which is placed at the substation and the receivers which are installed on trains. Therefore, the length of each channel changes according to the movement of the train. The whole length of each railway section is $25 \mathrm{Km}$. The frequencies from 1 to $30 \mathrm{MHz}$ are considered in this analysis.

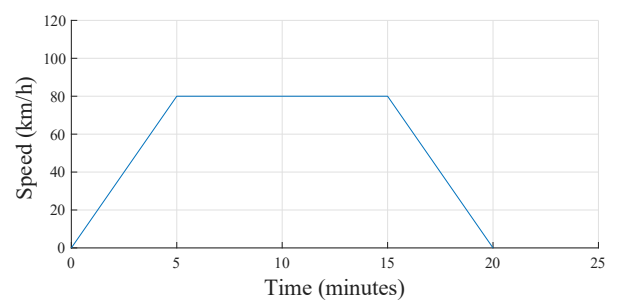

Fig. 7: Speed line-graph of section A train. 


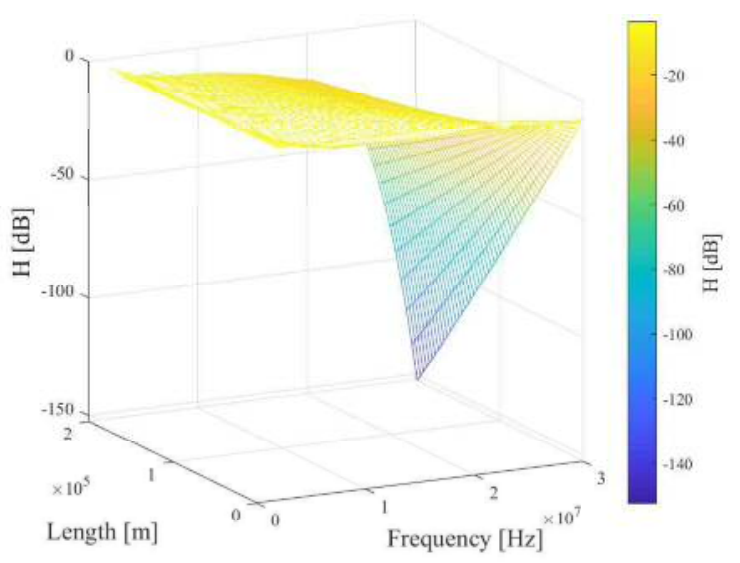

Fig. 9: A 3D surface plot for the channel response as a function of frequency and line length.

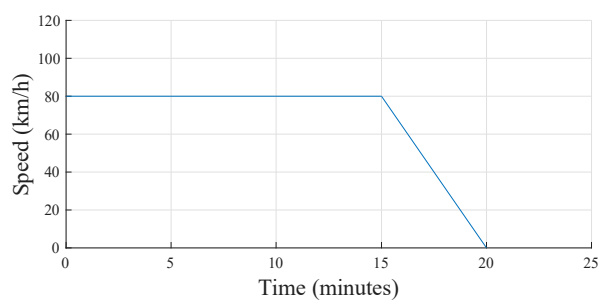

Fig. 8: Speed line-graph of section B train.

We first start with the train that moves within section A of the railway. It is assumed that this train moves away from the railway substation at different speeds as appears in Fig. 7. The train starts with steady-state then accelerates from speed zero up-to $80 \mathrm{Km} / \mathrm{h}$ in 5 minutes time. Then it continuous its $80 \mathrm{Km} / \mathrm{h}$ speed for 10 minutes before it starts to decelerate for 5 minutes from its current speed until it totally stops at the next train stop. Movement equations are used to calculate the train acceleration and to update the train position which represents the length of the channel.

The second scenario is the other train that moves towards the substation in section B of the railway. It is assumed that this train moves at different speeds towards the railway substation as shown in Fig. 8. The train first moves with $80 \mathrm{Km} / \mathrm{h}$ speed for 15 minutes. Then it decelerates for 5 minutes from its current speed until it reaches zero speed. The same movement equations that are used in the first scenario to calculate the train acceleration and to update the train position are used in this scenario. The train speed and position are updated every 0.25 second which represents $\mathrm{d} t$ in the movement equations.

A 3D surface for the channel response of the OLE in section A versus $l$ and $f$ is plotted in Fig. 9. It is obvious from this figure that both the length of OLE and the frequency have a negative impact on the transmission. As it can be seen that the transmission is less attenuated at lower frequencies and shorter distances. It is also clear that the point of the highest frequency and the furthest distance is the most attenuated one on the channel response curve.

To illustrate the nature of the OLE channel, the channel

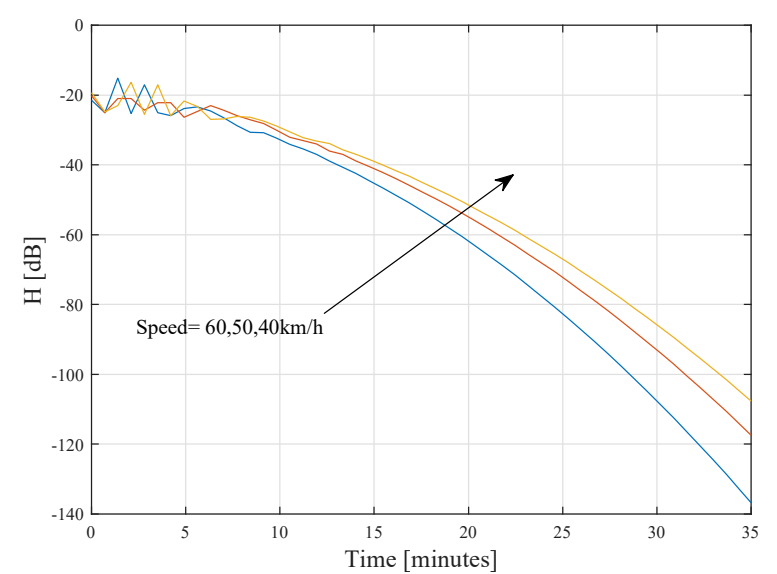

Fig. 10: Channel transfer function with respect to time traveling for different train speeds.

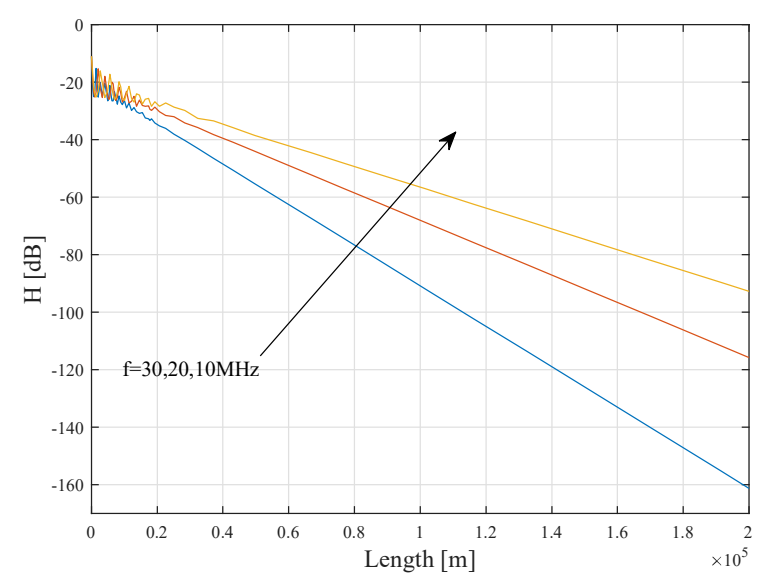

Fig. 11: Channel response versus distance for different frequencies.

response is shown as a function of the train traveling time in Fig. 10. It can be clearly seen from this figure that the attenuation of the transmitted signal becomes greater as the train traveling time increases. By having a closer look at this figure one can notice that the train speed can negatively affect the transmission. The higher the speed of the train the higher the attenuation of the transmission. However, the overall trend of attenuation increases towards the higher speeds and longer traveling time.

Fig. 11 shows the function of the OLE of section $\mathrm{A}$ as a function of the distance of the train for different values of frequencies. It is clear that the attenuation of the transmission increases when the train moves away from the substation. It is noticeable that when the train is still close to the substation, the transmission is less attenuated even for the higher frequencies. By looking at the three plotted curves of the frequencies, we can see that the lower the frequency the better the transmission is.

The channel response of section B of the railway is plotted versus the OLE length for different frequencies in Fig. 12. As anticipated, it can be seen that the signal strength increases when the train moves towards the substation which decreases the length of the OLE. On the other hand, the transmission is highly attenuated at the beginning of the train movement 


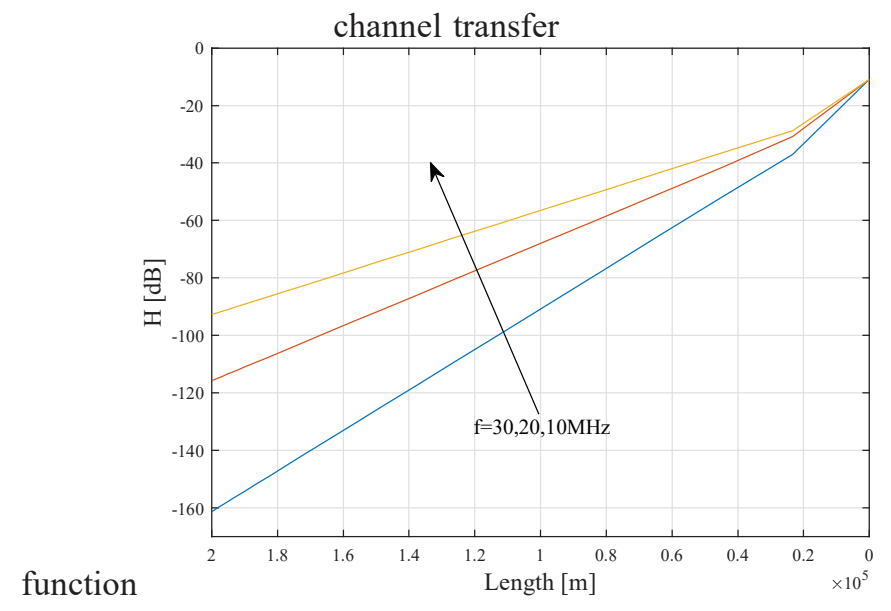

Fig. 12: Channel transfer function of the OLE of section B.

when the train is still far from the transmitter. However, the overall trend of attenuation decreases towards lower frequencies and shorter distances.

\section{Conclusions}

In this paper, two-port network model was developed to characterize the transfer function of the OLE channel. The BT electrification system of the railway was simplified and its equivalent circuit was derived. Furthermore, in order to update the position of the train, different movement equations that consider the acceleration and deceleration of the train were used. The results showed that the strength of the signal is negatively affected by the speed of the train, frequency and length of the OLE. Future work will include field measurements to compare the presented model to the actual channel of the OLE. Furthermore, the current model will be developed to include more than one train moving in one section of the railway.

\section{ACKNOWLEDGMENT}

This research has been jointly funded by OSL Rail Ltd, and the Faculty of Science and Engineering, Manchester Metropolitan University, UK.

\section{REFERENCES}

[1] R. D. White, "AC $25 \mathrm{kV} 50 \mathrm{~Hz}$ electrification supply design," in 5th IET Professional Development Course on Railway Electrification Infrastructure and Systems (REIS 2011), pp. 92-130, Jun 2011.

[2] A. Baxter, "Network rail a guide to overhead electrification," Feb. 2015.

[3] Jae-Jo Lee, Young-Jin Park, Soon-Won Kwon, Hui-Myong Oh, Haesoo Park, Kwan-Ho Kim, Dae-Young Lee, and Young-Hwa Jeong, "High data rate internet service over medium voltage power lines," in Int. Symp. Power Line Commun. and Its Appl.(ISPLC), 2005, pp. 405408, Apr. 2005.

[4] L. R. M. Castor, J. A. da Silva, and M. E. V. Segatto, "Medium voltage overhead power-line as a smart distribution grid for onshore oil gas industries automation and broadband data transport," in 2015 IEEE PES Innovative Smart Grid Technol. Latin America (ISGT LATAM), pp. 641-645, Oct. 2015.

[5] D. Lee, D. In, J. Lee, Y. Park, K. Kim, J. Kim, and S. Shon, "A field trial of medium voltage power line communication system for AMR and DAS," in 2009 Transmission Distrib. Conf. Exposition: Asia and Pacific, pp. 1-4, Oct. 2009.
[6] A. Cataliotti, D. Di Cara, and G. TinÃ ̌̌s "Model of line to shield power line communication system on a medium voltage network," in 2010 IEEE Instrum. Meas. Technol. Conf. Proceedings, pp. 14591462, May. 2010.

[7] M. Zimmermann and K. Dostert, "A multipath model for the powerline channel," IEEE Trans. Commun., vol. 50, pp. 553-559, Apr. 2002.

[8] T. Esmailian, F. R. Kschischang, and P. G. Gulak, "In-building power lines as high-speed communication channels: channel characterization and a test channel ensemble," Int. J. Commun. Syst., vol. 16, pp. 381400, 2003.

[9] W. Gheth, M. Rozman, K. M. Rabie, and B. Adebisi, "EMC measurements in indoor power line communication environments," in Int. Telecommun. Conf. (A. Boyaci, A. R. Ekti, M. A. Aydin, and S. Yarkan, eds.), (Singapore), pp. 189-200, Springer Singapore, 2019.

[10] A. Zupan, A. T. Teklic, and B. Filipovic-Grcic, "Modeling of $25 \mathrm{kv}$ electric railway system for power quality studies," in Eurocon 2013, pp. 844-849, Jul. 2013.

[11] B. Mellitt, C. J. Goodman, and R. I. M. Arthurton, "Simulator for studying operational and power-supply conditions in rapid-transit railways," Proc. of the Institution of Electrical Engineers, vol. 125, pp. 298-303, Apr. 1978 .

[12] C. J. Goodman, L. K. Siu, and T. K. Ho, "A review of simulation models for railway systems," in 1998 Int. Conf. Developments in Mass Transit Syst. Publ. No. 453), pp. 80-85, Apr. 1998.

[13] W. Mingli, C. Roberts, and S. Hillmansen, "Modelling of AC feeding systems of electric railways based on a uniform multi-conductor chain circuit topology," in IET Conf. Railway Traction Syst. (RTS 2010), pp. 1-5, Apr. 2010.

[14] M. Plakhova, "Implementation of the derivative-based technique for solving a $2 * 25 \mathrm{kv}$ AC bivoltage traction system," pp. 1-7, Dec. 2015.

[15] Y. Tanaka, M. Ishikawa, and K. Kurogi, "Study for electromagnetic inference under construction in boosting transformer feeding system," in 2016 19th Int. Conf. Electr. Machines and Syst. (ICEMS), pp. 1-4, Nov. 2016.

[16] J. R. Tierney and R. J. Turner, "Improvement to the booster transformer/return conductor method of suppressing $50 \mathrm{~Hz}$ interference from A.C.-electrified railway systems," IEE Proc. B - Electr. Power Appl., vol. 128, pp. 61-66, Jan. 1981.

[17] E. Pilo, L. Rouco, and A. Fernandez, "Catenary and autotransformer coupled optimization for $2 \times 25 \mathrm{kv}$ systems planning," vol. 88, pp. 747756, Jone. 2006

[18] E. Pilo, L. Rouco, A. Fernandez, and L. Abrahamsson, "A monovoltage equivalent model of bi-voltage autotransformer-based electrical systems in railways," IEEE Trans. Power Del., vol. 27, pp. 699-708, Apr. 2012.

[19] A. Dolara and S. Leva, "Calculation of rail internal impedance by using finite elements methods and complex magnetic permeability," Int. J. Veh. Technol., vol. 2009, Jan. 2009. 